\title{
Evidence of Resonant Mode Coupling in the Hot B Subdwarf Star KIC 10139564
}

\author{
W. Zong ${ }^{1, a}$, S. Charpinet ${ }^{1}$ and G. Vauclair ${ }^{1}$ \\ ${ }^{1}$ CNRS, Université de Toulouse, UPS-OMP, IRAP, Toulouse 31400, France
}

\begin{abstract}
The Kepler spacecraft provides new opportinuties to observe long term frequency and amplitude modulations of oscillation modes in pulsating stars. We analyzed more than three years of uninterrupted data obtained with this instrument on the hot B subdwarf (sdB) star KIC 10139564 and found clear signatures of nonlinear resonant mode coupling affecting several multiplets. The observed periodic frequency and amplitude modulations may allow for new asteroseismic diagnostics, providing in particular ways to measure linear growth rates of pulsation modes in hot subdwarf stars for the first time.
\end{abstract}

\section{Introduction}

The Kepler spacecraft is a magnificent instrument to observe long term frequency and amplitude modulations of oscillation modes in pulsating stars. Among the 18 oscillating sdB stars monitored in the Kepler field, KIC 10139564 is the unique p-mode dominated (V361 Hya type) pulsator with low amplitude g-mode oscillations. It has been observed by Kepler for 38 months in short cadence (SC) mode without interruption. Its rotation period is $\sim 26$ days according to several multiplets with common frequency spacings found in both the g- and p-mode regions. Thus, it is a well suited candidate to investigate resonant mode coupling mechanisms that could affect amplitudes and frequencies of oscillation modes - in particular those in rotationally split multiplets - over long timescales. The theory for these mechanisms has been investigated long before the era of space observations but suffered from the lack of observational evidence and constraints due to the timescales involved. We present the analysis of KIC 10139564 showing that some multiplets (in particular triplets) clearly have amplitudes and frequencies varying with time in a manner that can be related to nonlinear resonant mode coupling. Some of these modes would be in the intermediate regime of a resonant coupling mechanism occuring when linear frequencies follow the relation $v_{1}+v_{2} \sim 2 v_{0}$, which generally happens for frequencies split by slow stellar rotation [1]. The characterization of the induced modulations may offer new asteroseismic diagnostics, providing in particular ways to measure linear growth rates of pulsation modes in hot subdwarf stars for the first time.

\section{Results and Discussion}

The sdB star KIC 10139464 has been continuously observed by Kepler since quarter Q5.1 up to Q17.2. The high precision photometric data cover $~ 1,147.5$ days (38 months), with a duty circle of $\sim 89 \%$. We used a dedicated software, FELIX, to extract the frequencies from the lightcurve (details of this program can be found in Ref [2]). We defined a filter window covering 200 days and slid the filter window along the whole light curve by time steps of 20 days, thus constructing a time-frequency diagram. We also extracted the frequencies "chunk by chunk", i.e Â., the 38-month light curve of

a e-mail: weikai.zong@irap.omp.eu

This is an Open Access article distributed under the terms of the Creative Commons Attribution License 4.0, which permits unrestricted use, distribution, and reproduction in any medium, provided the original work is properly cited. 

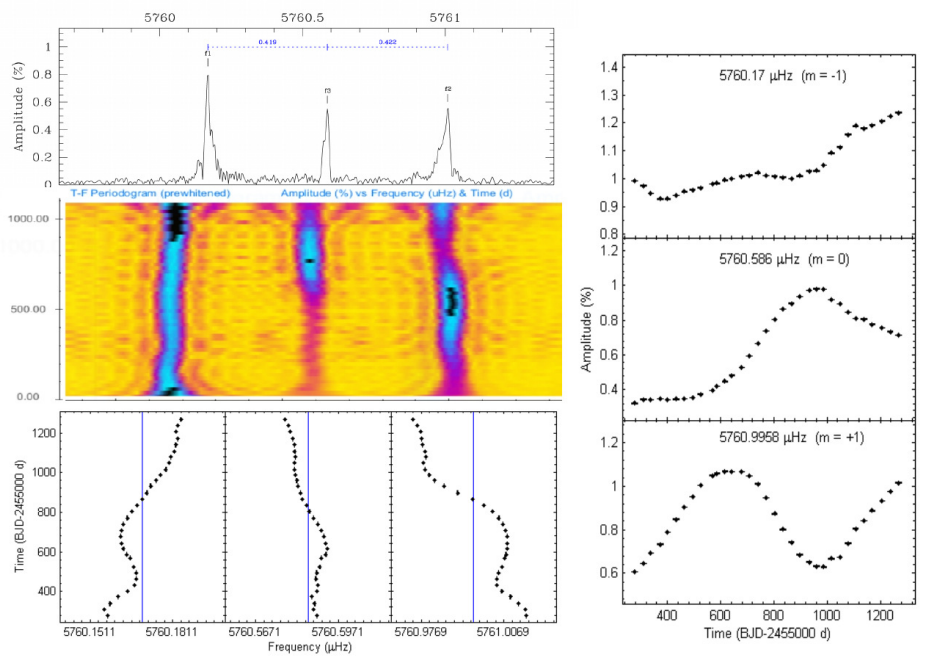

Fig. 1. Frequency and amplitude modulations of the dominant triplet near $5760 \mu \mathrm{Hz}$.

KIC 10139564 was divided into 33 chunks. Each chunk contains 9-month of SC mode data, except the last three chunks being at the end of the Kepler observations.

The amplitude and frequency modulations of the dominant triplet at $5760 \mu \mathrm{Hz}$ are shown in Fig. 1 . The right panel shows the amplitude modulations of each component forming the triplet. The frequencies in the two lower-left panels also show clear signatures of periodic modulations. These modulations can be related to nonlinear resonant mode coupling mechanisms in pulsating stars. This triplet at $5760 \mu \mathrm{Hz}$ at least qualitatively behaves like if it is in the intermediate regime of the resonance, in which the oscillation modes undergo periodic amplitude and frequency modulations with a time scale of roughly a few times the inverse of the growth rate of the pulsating mode [3]. Therefore periodic modulations as found in the illustrated triplet could in principle be used to measure the growth rate. Other multiplets detected in KIC 10139564 show similar behaviors not presented here due to space constraints.

\section{Conclusion}

Frequency and amplitude modulations of oscillation modes have been found in several rotationally split multiplets detected in the sdB pulsator KIC 10139564, thanks to the high quality and long duration photometric data obtained with the Kepler spacecraft. These modulations show signatures pointing toward nonlinear resonant coupling mechanisms occuring among the multiplet components. This is the first time that such signatures are identified in $\mathrm{sdB}$ pulsating stars. Periodic modulations of frequency and amplitude that occur in the intermediate resonant regime may allow for new asteroseismic diagnostics, providing in particular a way to measure for the first time linear growth rates of pulsation modes in sdB stars. These growth rates are directly linked to the mode driving engine, a $\kappa$-mechanism involving the iron bump and powered up by radiative levitation. Such results should motivate further theoritical work on nonlinear resonant mode coupling mechanisms and revive interest in nonlinear stellar pulsation theory in general.

\section{References}

1. Buchler, J. R., Goupil, M.-J. \& Serre, T. A\&A, 295, (1995), 405

2. Charpinet, S., Green, E. M., Baglin, A., et al. A\&A, 516, (2010), L6

3. Goupil, M. J., Dziembowski, W. A. \& Fontaine, G. BaltA, 7, (1998), 21 